\title{
Three Dimensions of Association Link Migraine Symptoms and Functional Connectivity
}

2 Samuel R. Krimmel ${ }^{1,2}$, Danielle D. DeSouza ${ }^{3}$, Michael L. Keaser ${ }^{1}$, Bharati M. Sanjanwala ${ }^{3}$, Robert P.

3 Cowan $^{3}$, Martin A. Lindquist ${ }^{4}$, Jennifer Haythornthwaite ${ }^{5}$, David A. Seminowicz ${ }^{1^{*}}$

$4 \quad{ }^{1}$ Department of Neural and Pain Sciences, School of Dentistry, and Center to Advance Chronic Pain

5 Research, University of Maryland, Baltimore, MD, 21201, USA.

$6{ }^{2}$ Program in Neuroscience, University of Maryland School of Medicine, Baltimore, MD, 21201 USA.

$7 \quad{ }^{3}$ Department of Neurology and Neurological Sciences, Headache and Facial Pain Program, Stanford

8 University, CA, 94305, USA.

$9{ }^{4}$ Department Biostatistics, Johns Hopkins Bloomberg School of Public Health, Baltimore, MD, 212015, 10 USA.

$11{ }^{5}$ Department of Psychiatry and Behavioral Sciences, Johns Hopkins University School of Medicine, 12 Baltimore, MD, 21224, USA.

\section{Abstract}

Migraine is a heterogeneous disorder with variable symptoms and responsiveness to therapy.

24 frequency, pain catastrophizing, affect, sleep disturbances, and somatic abnormalities, and was

27 change from baseline to post-intervention - were associated with clinical responsiveness to mind-body 
default mode network connectivity. The final dimension was related to pain catastrophizing, and salience, sensorimotor and default mode network connectivity. These unique symptom/brain-mappings over three dimensions provide novel network targets to modify specific ensembles of symptoms. In addition to performing CCA, we evaluated the current clustering of migraine patients into episodic and chronic subtypes, and found no evidence to support this clustering. However, when using RSFC scores from the three significant dimensions, we identified a novel clustering of migraine patients into four biotypes with unique functional connectivity patterns. These findings provide new insight into individual variability in migraine, and could serve as the foundation for novel therapies that take advantage of migraine heterogeneity.

Keywords: chronic pain, networks, individual variability, pain catastrophizing, mindfulness-based stress reduction, functional MRI, treatment

\section{Introduction}

Migraine is a common, poorly managed, and disabling disorder, affecting over $10 \%$ of the global population (Leonardi \& Raggi, 2013; Lipton et al., 2001, 2007; Rasmussen, 1995). Even with improved treatments (Edvinsson et al., 2018; Goadsby et al., 2017; Seminowicz et al., 2020; Yuan et al., 2019), it is clear that continued research is warranted. While its diagnosis is based on migraine attacks, migraine is a much more complex disorder, often featuring sleep, affective, cognitive, and general health

47 abnormalities (Breslau et al., 1994, 2000; Karthik et al., 2012; Lin et al., 2016). Importantly, the presentation of these symptoms is heterogeneous across subjects, which is an important consideration

49 for migraine research and treatment. Improved understanding of this variability would allow for precision medicine, and would also help to stratify migraine patients into possible subcategories. 
The brain can be functionally segregated into structured patterns of covariance, called networks

52 (Yeo et al., 2011), and these brain networks have been linked to migraine symptoms (Schwedt et al.,

53 2015). A common method for identifying brain networks is resting state functional connectivity (RSFC), a

54 measure of functional coupling between regions of the brain (Mclntosh, 2000), typically estimated

55 through correlating timeseries from functional units in functional magnetic resonance imaging (fMRI)

56 acquired during rest (i.e., in the absence of a task or stimulus). These studies typically associate RSFC

57 with individual migraine symptoms, but do not take into account that symptoms are interdependent, as

58 are estimates of RSFC. psychopathology (Drysdale et al., 2017; Mihalik et al., 2019; Xia et al., 2018), and mind-wandering (H.-T.

67 standard mass univariate analyses, these 3 dimensions were each informed by many clinical and RSFC

68 features simultaneously. These dimensions related to symptoms globally and also to more specific

69 combinations of symptoms, and reveal potential targets for brain stimulation or other interventions.

71 mindfulness-based stress reduction (MBSR+), an emerging non-pharmacologic therapy for chronic pain.

72 Finally, we were able to show that the current clustering of migraine into episodic or chronic subtypes

73 based on headache frequency alone is not supported, and propose a new data driven biotyping of

74 migraine patients into 4 clusters that is based on RSFC and symptoms together. 
Methods

Overview

We took clinical

measures and RSFC from

two study centers. 143

subjects had complete

RSFC and clinical data, and

were used as input data for

the CCA. 166 subjects had

complete clinical data and

were used to assess the

empirical support of

episodic versus chronic

subtypes of migraine.

Multi-site harmonization

was used to correct for

effects of site For RSFC,

while still maintaining

clinical information. We first

applied PCA to both clinical

and RSFC data to address

multicollinearity while

reducing dimensionality.

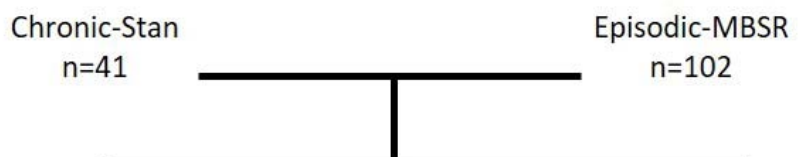

$\downarrow$

Headache frequency
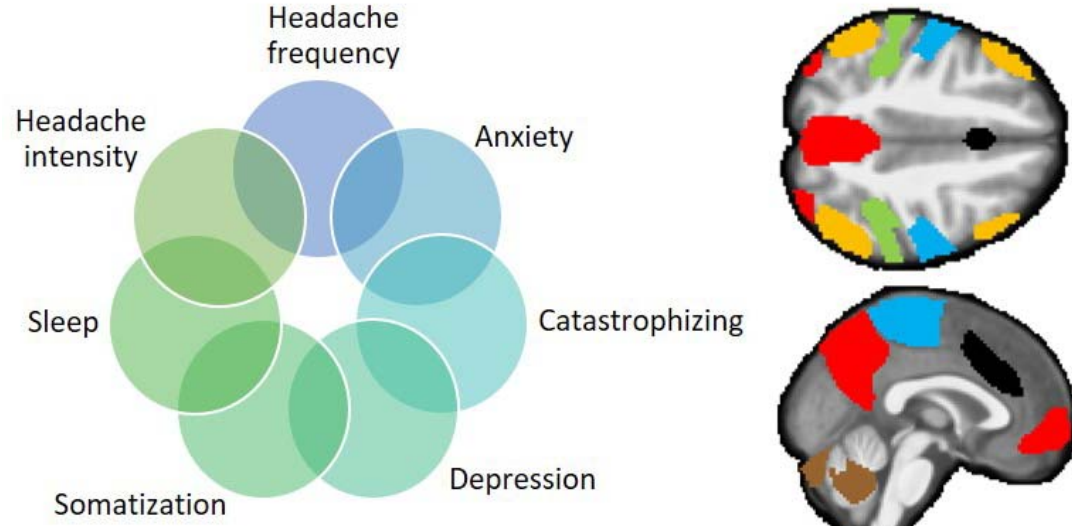

Cerebellar Frontoparietal Dorsal attention Salience Sensory Default mode

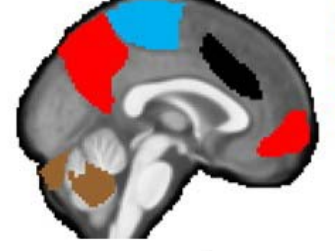

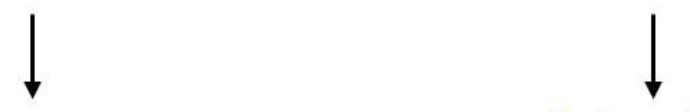

Feature selection; site harmonization; PCA

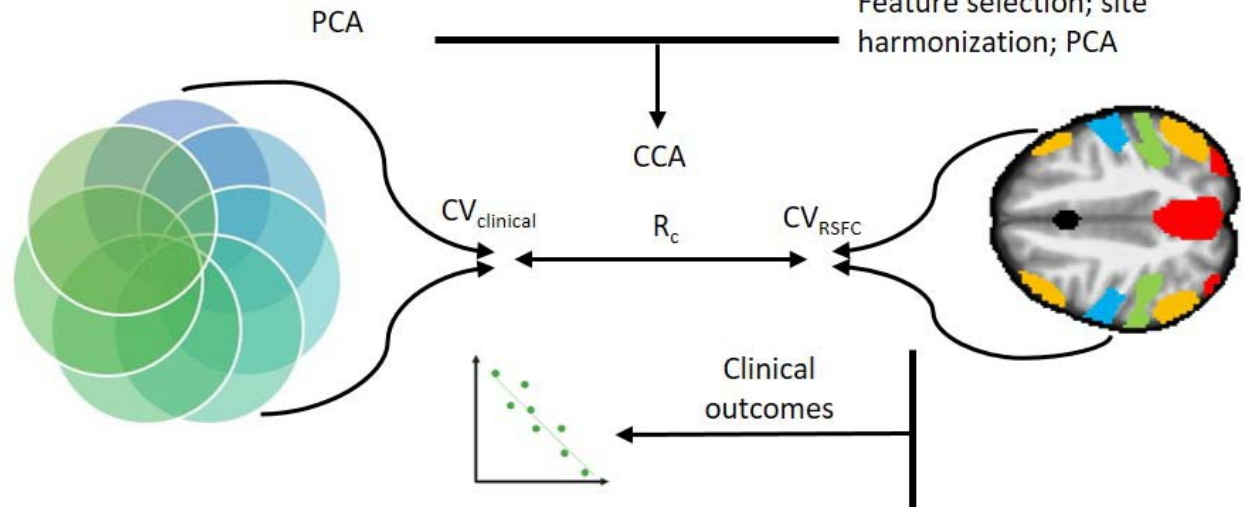

Clustering

Fig 1. Study overview. For Canonical Correlation Analysis (CCA), 143 migraine subjects were collected from two sites. Seven clinical measures were acquired along with resting-state functional connectivity (RSFC) estimates from 24 regions spanning six functional networks. After site harmonization of RSFC and principal components analysis (PCA), CCA was used to associate clinical and biological data. CCA is used to study the relationship between variables from two domains of interest measured in one sample. In CCA, linear combinations of the input variables from both domains are created (called canonical variates) so that the correlation between the combined variables (canonical correlation, $\mathrm{R}_{\mathrm{c}}$ ) is maximized. RSFC canonical variates were associated with clinical outcomes to mind-body therapy and underwent clustering analysis to reveal biotypes of migraine. $P C A=$ principal components analysis; $R_{c}=$ canonical correlation; $\mathrm{CV}_{\mathrm{RSFC}}=$ canonical variates for $\mathrm{RSFC} ; \mathrm{CV}_{\text {clinical }}=$ canonical variates for clinical data. 
Regularized CCA was then performed on components explaining $80 \%$ of variance and permutation tests were used to assess statistical significance. Canonical correlation is simply a correlation between canonical variates, where canonical variates are created from two domains of data (clinical and RSFC) so that the canonical correlation is maximized. Stated differently, CCA allows for dimensions of association to be found between clinical and RSFC, where these associations are formed through unique combinations of clinical and RSFC variables. Each canonical correlation consists of a clinical and RSFC canonical variate that are created to be associated with one another. For significant canonical correlations, RSFC canonical variates were then tested for association with motion (a major confound for RSFC studies) and clinical improvement from mind-body therapy. Finally, these scores underwent kmeans clustering to identify biotypes in our sample (see Fig 1 for study overview).

Participants

Data from two migraine neuroimaging studies were analyzed, referred to here as the chronic-

Stanford and episodic-UMB datasets. In the chronic-Stanford dataset, participants were recruited to participate in research investigating biomarkers of chronic daily headache using clinical, behavioral, and magnetic resonance imaging (MRI) data at Stanford University. All subjects were over 18 years of age and met the International Classification of Headache Disorders ICHD-3 diagnostic criteria for chronic migraine as determined by a physician for a minimum of three months (International Headache Society, 2018). Patients were excluded if they had any MRI contraindications or a history of severe neurologic/psychiatric disorders. Subjects provided written informed consent in accordance with

117 Stanford University guidelines. MRI scans used in this analysis were a structural T1-weighted scan 118 (repetition time [TR] $5900 \mathrm{~ms}$, echo time [TE] minimum, flip angle $15^{\circ}$, voxel size $0.9 \times 0.9 \times 1 \mathrm{~mm}$ ), and 119 an eyes closed resting state functional scan (8 minutes, gradient echo spiral-pulse, TR 2000, TE 30 ms, 120 slice thickness $4 \mathrm{~mm}$, FOV $220 \mathrm{~mm}$, flip angle $80^{\circ}$, voxel size $3.4 \times 3.4 \times 4.5 \mathrm{~mm}$ ). For analyses of clinical 121 data only, subjects were excluded due to missing data, leaving a total of 46 subjects for analysis (average 
122

123

124

age of 39 , s.d. $=13,38$ female). For MRI analyses, subjects were excluded due to poor MRI quality, excessive motion during resting state (average framewise displacement greater than $0.4 \mathrm{~mm}$ ), and missing clinical data, leaving a total of 41 subjects for analysis (average age of 38, s.d. $=13,34$ women).

The second dataset, episodic-UMB, consisted of data from a previously described clinical trial assessing enhanced mindfulness-based stress reduction (MBSR+) treatment of migraine (Seminowicz et al., 2020). Briefly, participants were between the ages of $18-65$ and met the ICHD-3 criteria for episodic migraine with or without aura for more than a year (International Headache Society, 2018). Participants were excluded if they had a history of mindfulness training, reported severe or unstable psychiatric symptoms, and/or used opioids. All patients provided written informed consent in accordance with University of Maryland Baltimore standards. MRI scans used in this analysis were a structural T1weighted scan (TR 2300ms, TE $2.98 \mathrm{~ms}$, slice thickness $1 \mathrm{~mm}$, FOV $256 \mathrm{~mm}$, flip angle $9^{\circ}$, and voxel size 1 $\times 1 \times 1 \mathrm{~mm}$ ) and an eyes open resting state scan (10 minutes, echo planar imaging, TR $2000 \mathrm{~ms}$, TE 28 $\mathrm{ms}$, slice thickness $4 \mathrm{~mm}$, FOV $220 \mathrm{~mm}$, flip angle $77^{\circ}$, and voxel size $3.4 \times 3.4 \times 4 \mathrm{~mm}$ ). For analyses of clinical data only, subjects were excluded due to missing data, leaving a total of 120 subjects for analysis (average age of 38, s.d. $=12,106$ female). For analyses of MRI data, subjects were excluded due to poor MRI quality, excessive motion during resting state (average framewise displacement greater than 0.4 $\mathrm{mm}$ ), and missing clinical data, for a total of 102 subjects (average age of $38, \mathrm{s.d} .=12,91$ women). 83 of these subjects were also randomized to a clinical trial receiving either MBSR+ $(n=42)$ or an active control condition (stress management for headache; $n=41$ ). These randomized participants received additional scans 10 and 20 weeks after the baseline scan (Seminowicz et al., 2020). Two MBSR+ subjects were excluded when analyzing changes in RSFC canonical variate scores leaving 40 subjects. 


\section{Magnetic Resonance Imaging Preprocessing}

Preprocessing was performed using FMRIPREP version stable (Esteban et al., 2020), a Nipype

(Gorgolewski et al., 2017) based tool. Each T1-weighted (T1w) volume was corrected for INU (intensity non-uniformity) using N4BiasFieldCorrection v2.1.0 (Tustison et al., 2010) and skull-stripped using antsBrainExtraction.sh v2.1.0 (using the OASIS template). Spatial normalization to the ICBM 152 Nonlinear Asymmetrical template version 2009c (Fonov et al., 2009) was performed through nonlinear registration with the antsRegistration tool of ANTs v2.1.0 (Avants et al., 2008), using brain-extracted versions of both T1w volume and template. Brain tissue segmentation of cerebrospinal fluid (CSF), white-matter (WM) and gray-matter (GM) was performed on the brain-extracted T1w using fast (FSL v5.0.9 (Zhang et al., 2001)).

Functional data was slice time corrected using 3dTshift from AFNI v16.2.07 (Cox, 1996) and motion corrected using mcflirt (FSL v5.0.9 (Jenkinson et al., 2002)). This was followed by coregistration to the corresponding T1w using boundary-based registration (Greve \& Fischl, 2009) with six degrees of freedom, using flirt (FSL). Motion correcting transformations, BOLD-to-T1w transformation and T1w-to-template ( $\mathrm{MNI}$ ) warp were concatenated and applied in a single step using antsApplyTransforms (ANTs v2.1.0) using Lanczos interpolation.

Frame-wise displacement (Power et al., 2014) was calculated for each functional run using the implementation of Nipype. ICA-based Automatic Removal Of Motion Artifacts (AROMA) was used to generate aggressive noise regressors as well as to create a variant of data that is non-aggressively denoised (Pruim et al., 2015). Many internal operations of FMRIPREP use Nilearn (Abraham et al., 2014), principally within the BOLD-processing workflow. For more details of the pipeline see https://fmriprep.readthedocs.io/en/stable/workflows.html. 


\section{Resting State Functional Connectivity Processing}

Resting-state denoising and seed-based analysis were conducted in the CONN functional connectivity toolbox version $19 f$ (Whitfield-Gabrieli \& Nieto-Castanon, 2012;

http://www.nitrc.org/projects/conn). Given sustained controversy concerning global signal, we elected not to remove global signal in our analyses (Murphy \& Fox, 2017). We performed aCompCor (Behzadi et al., 2007; Muschelli et al., 2014) to remove noise captured in white matter and CSF. We calculated the first five eigenvectors from four erosions of subject specific white matter masks and two erosions of subject specific CSF masks, as these levels of erosion no longer contain global signal (Power et al., 2017). To avoid noise being reintroduced while removing frequency specific noise, voxel-wise and regressor data were simultaneously band-pass filtered $(0.008-0.15 \mathrm{~Hz})$ and all data underwent linear detrending. Following resting state functional denoising, timeseries were extracted from 32 ROls based on an existing independent component analysis of the Human Connectome Project available from the CONN functional connectivity toolbox. These ROls were organized into default mode, sensorimotor, visual, salience/cingulo-opercular, dorsal attention, frontoparietal, language and cerebellar networks. To estimate functional connectivity, the $32 \mathrm{ROI}$ timeseries were correlated and the resulting Pearson correlation coefficient were converted to $\mathrm{z}$ scores. We excluded functional connections with visual and language network seeds, as we did not believe that these would be associated with the clinical symptoms in our analysis. Language network regions included parts of the posterior superior temporal gyrus and the inferior frontal gyrus (consistent with Wernicke's and Broca's area). Visual network regions included nodes in the occipital cortex. While the occipital cortex has been implicated in migraine, it is often associated with symptoms of aura (Hadjikhani et al., 2001), a symptom domain that was not measured in our two datasets. This left a total of 24 ROls with 276 unique functional connections. 
Study site has been shown to influence fMRI data and should be adjusted for when possible

(Noble et al., 2017; Van Horn \& Toga, 2009; Yu et al., 2018). To adjust for effects of site while controlling

for clinical variables, age, and sex, we used Combining Batches of microarray data (ComBat) (Johnson et

\section{Clinical Features}

Participants completed multiple self-report questionnaires to assess clinical characteristics. The

with headaches. We also examined the exaggerated mental set applied to pain or anticipation of pain

204 (Spitzer et al., 2006) and the patient health questionnaire 9 (PHQ-9) measure of depression (Kroenke et

\section{Principal Components Analysis}

We used Principal Components Analysis (PCA) to address multicollinearity and to reduce the

208 dimensionality of our data. PCA creates a set of orthogonal components that can be linearly combined

209 to explain all of the variance in the original dataset. We used singular value decomposition and selected 
211 first 35 RSFC components, explaining more than 80 percent of the RSFC variance (Fig 2B). These

212 components were used as input data for the canonical correlation analysis (see below section:

213 Regularized Canonical Correlation Analysis).

CCA is a method for identifying multivariate relationships between sets of variables (Hotelling,

Harold, 1935). It is used to study the relationship between variables from two domains of interest (e.g.,

the correlation between the combined variables (canonical correlation, $R_{c}$ ) is maximized. Similar to PCA 
unique canonical weights and 100 corresponding testing sets to evaluate performance. For our final

236 model, we used the regularization parameters that yielded the best average $R_{c}$ value in held out testing

237 data for the first canonical correlation.

CCA was performed following PCA of clinical and RSFC data. However, the interpretation of the

rotation matrix is challenging, and we therefore transformed the CCA weights back into their original

space using the CCA model weights and the rotation matrix. Following this transformation, we were able

242 to use the CCA model weights in the original clinical and RSFC spaces. Applying these weights to clinical

243 and RSFC data yielded clinical and RSFC canonical variates respectively. We did not use these

244 reconstructed weights directly for interpretation, and instead used canonical loadings for the purposes

245 of interpretation (see below section: Statistics and figure 4). We did however use these weights to

246 extract canonical variates over time.

We examined if the canonical variates obtained from CCA were associated with outcomes to

$249 \mathrm{MBSR}+$ in the episodic-UMB dataset. To do this, we examined subjects randomized to the enhanced

250 mindfulness-based stress reduction condition and correlated baseline canonical variates for RSFC with

251 the reduction in headache frequency after 20 weeks, the primary outcome of the trial (Seminowicz et

252 al., 2020). We also sought to determine if changes in canonical variates were associated with reductions

253 in headache frequency. To do this, we calculated the canonical variates using model weights from the

254 CCA model described above, and calculated difference scores before and after therapy (20-week gap in

255 between scans). In this analysis we did not use ComBat corrected RSFC data when determining changes

256 in canonical variate scores, since the data were from a single site. The relationship between the change 
in canonical variate scores and reductions in headache frequency was assessed by means of correlation

258 analysis over the corresponding time period.

Determining Support for Episodic vs Chronic Migraine Clustering on Migraine Symptoms

Chronic versus episodic migraine can be thought of as a clustering solution to migraine and is clustering solution was supported by a more holistic catalogue of clinical data. We therefore determined the silhouette index for episodic versus chronic distinction using seven clinical variables in the combined sample ( $n=166$ ). Should the episodic/chronic clustering have a silhouette index no better than chance (i.e., likely coming from a multivariate normal distribution), then is would argue against the current field norm of episodic and chronic migraine subtypes (see below section: Statistics).

We sought to identify biotypes in migraine based on the significant RSFC canonical variates from CCA using k-means clustering. We used Euclidean distance as our distance metric for all clustering. We compared 26 clustering metrics using the NBclust package (Charrad et al., 2014) with cluster number (k) ranging from 2 to 10 . For each value of $k$, a score for all 26 clustering metrics was computed, and cluster

272 bperformance was ranked per metric (e.g., the value of $k$ with the largest silhouette index received a

273 rank of one). Per metric, a value of $k$ 'won' if it received the first rank and therefore had the best

274 performance for that specific clustering metric. We chose a final value for $k$ using a majority rule, where

275 the cluster number that had the most total victories (came in first for a clustering metric) was deemed

276 the best overall. We paid special attention to the silhouette index, as this metric has been shown to

277 accurately identify clusters of data (Petrovic, 2006). 


\section{Statistics}

To determine statistical significance of our CCA we performed a permutation test by constructing 5000 bootstraps with replacement (Efron, 2000) using the final model lambda parameters (see Results section: Three Modes of Association Were Found Between RSFC and Migraine Symptoms). For each bootstrap, we calculated $R_{c}$ values for four canonical correlations and then compared this null distribution against true $R_{c}$ values using a one-sided t-test for each of the four canonical correlations in a process previously described (Dinga et al., 2019). To interpret CCA results we calculated canonical loading by correlating canonical variates with their respective model inputs (e.g., RSFC values). We used a false discovery rate multiple comparisons correction on the resultant $p$ values (Benjamini et al., 2001). Determining significance for clustering analysis can be challenging (Dinga et al., 2019; Liu et al., 2008). For our clustering, we tested the true silhouette index scores for the final clustering solution relative to 5000 multivariate Gaussian distributions based on the mean and covariance of the input data using a one sample t-test, in a process similar to ones previously described (Dinga et al., 2019; Liu et al., 2008). This approach allows us to test against the null model of a multivariate normal distribution which does not support clustering. A Spearman rank correlation was used for all correlation analysis unless stated otherwise. For all analyses, significance was determined using a 0.05 FDR-corrected threshold.

\section{Results}

Three Modes of Association Were Found Between RSFC and Migraine Symptoms

We used CCA to identify dimensions of covariance between a diverse array of clinical symptoms and RSFC. We optimized lambda values for regularization using performance in held out testing data to maximize canonical correlation $\left(R_{c}\right)$ for the first pair of canonical variates. The best performance in testing data for the first canonical correlation was an average $R_{c}=0.6$ with lambda values of 0.3 for clinical data and 0.9 for RSFC, and we used these regularization parameters for our final model. In the 
302 final model, the first canonical correlation was $R_{c}=0.71, N=143, p=0.0002$ (Fig 2C, D), the second was

$303 R_{c}=0.49, N=143, p=0.0023(F i g 1 E, F)$, the third was $R_{c}=0.43, N=143, p=0.006$ (Fig $\left.1 G, H\right)$, and the

304 fourth was $R_{c}=0.35, N=143, p=0.098$. The average $R_{c}$ values for held-out testing data is as follows: first

305 canonical correlation $R_{c}=0.6$, s.d. $=0.19$, second $R_{c}=0.14$, s.d. $=0.25$, third $R_{c} 0.19$, s.d. $=0.23$, and

306 fourth $R_{c}=0.03$, s.d. $=0.23$. We focused interpretation and further analysis on the first three significant

307 canonical correlations. Note that each canonical correlation is between a pair of normally distributed

308 clinical and RSFC canonical variates. Therefore, from the three significant canonical correlations, each

309 subject has 3 RSFC and 3 clinical canonical variates. 

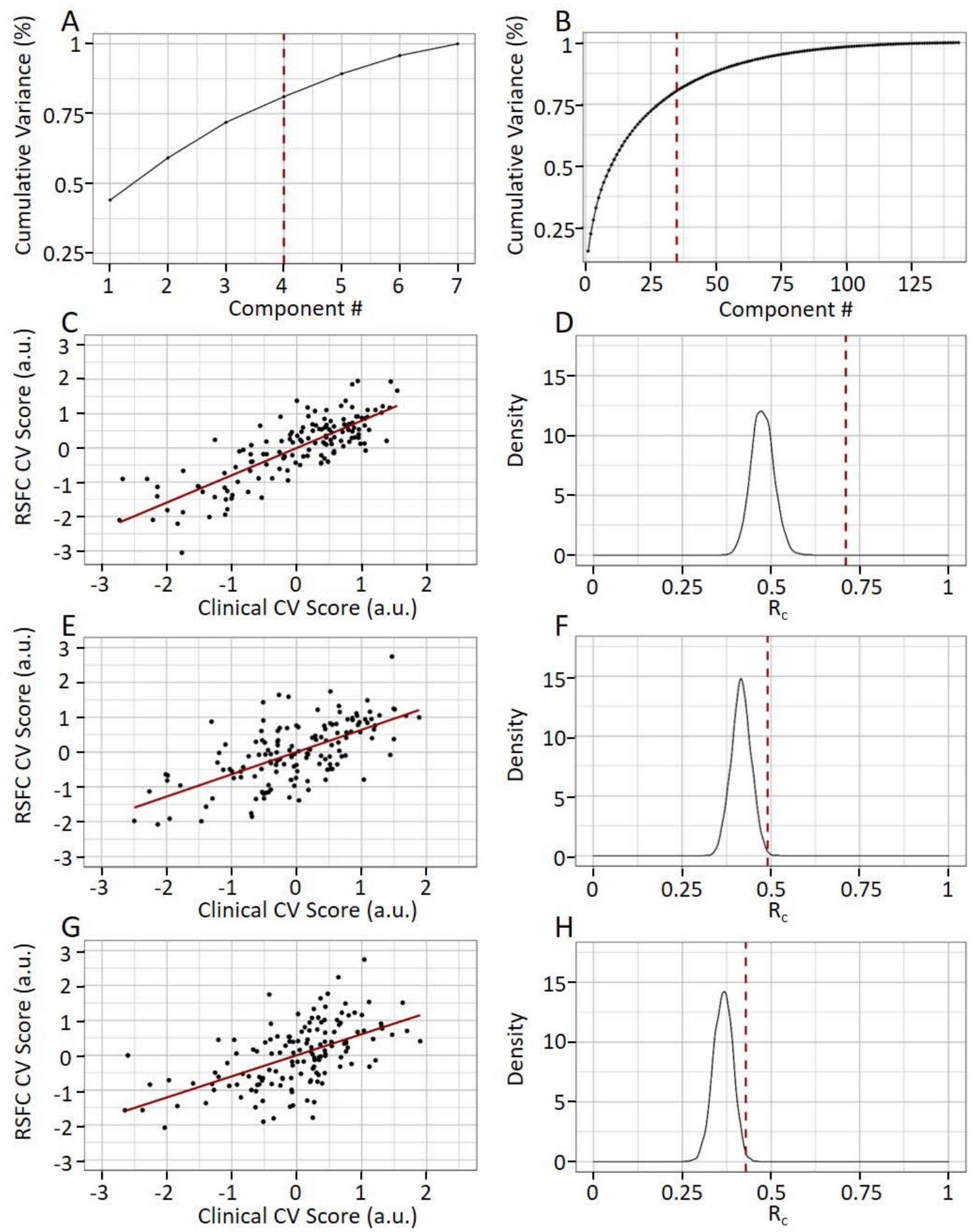

Fig 2. Using CCA to reveal three dimensions of association between clinical features and RSFC. Cumulative variance explained from clinical (A) and RSFC (B) principal components. Red lines indicate $80 \%$ variance explained. Canonical correlation ( $R_{c}$ ) between RSFC and clinical canonical variates for first $(C)$, second $(E)$, and third $(G)$ canonical correlations. Permutation test for significance of $R_{c}$ value (shown in red) relative to 5000 permutations for first (D), second (F), and third $(H)$ canonical correlations. $R_{c}=$ canonical correlation; $\mathrm{CV}=$ canonical variate. 
Association of RSFC Canonical Variates and Scanner Motion

312

Given that motion has profound effects on estimates of RSFC (Power

313

314

315

316

317

318

319

320

321

322

323

324

325

326

327

328

329

330

331

332

333

et al., 2012), we ensured that the canonical variates for RSFC were

unrelated to motion. We did not find a significant correlation

between average framewise displacement during the resting state

scan with the first $r_{s}=0.12, N=143, p=0.14($ Fig $3 A)$, second $r_{s}=$

$0.08, N=143, p=0.3\left(\right.$ Fig 3B), nor third $r_{s}=-0.04, N=143, p=0.6$

(Fig 3C) RSFC canonical variates. We therefore concluded that there

was no evidence for a relationship between RSFC canonical variates

and subject movement.

Interpretation of CCA Model

We correlated canonical variates with their respective

RSFC/clinical symptoms (i.e., canonical loadings) to interpret the

CCA model. While each of the three resultant dimensions consisted

of clinical and RSFC canonical variates, we chose to name each

dimension with a clinically derived name for ease of discussion. The

first canonical variate for symptoms correlated in the same direction

with all 7 clinical features (Fig 4A). Hence, we interpret the first

clinical canonical correlation as the 'global symptom dimension'.

Patients with positive canonical variate values for the first canonical

correlation had globally better symptoms (less severe symptoms

overall). The associated first RSFC canonical variate primarily

reflected frontoparietal network and dorsal attention network connectivity (Fig 4B).
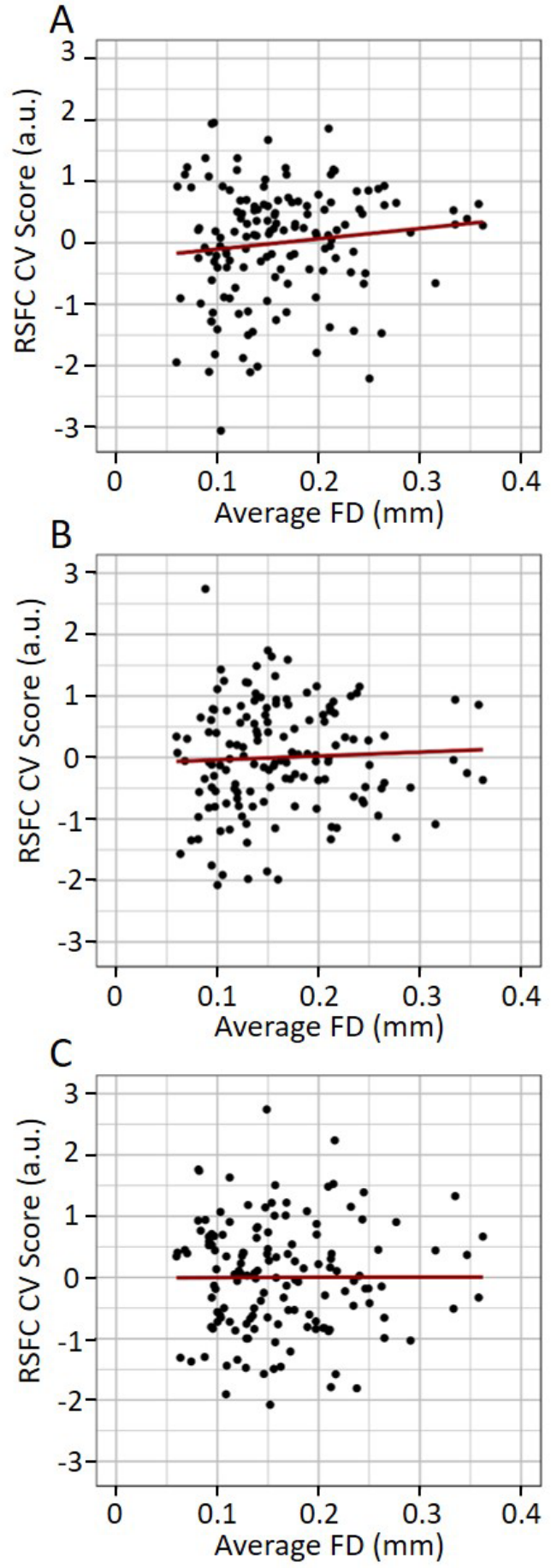

Fig 3. No association between canonical variates and subject motion. Scatter plot for first (A), second (B), and third (C) RSFC canonical variate relationship with average framewise displacement. $C V=$ canonical variate, $\mathrm{FD}=$ framewise displacement. 


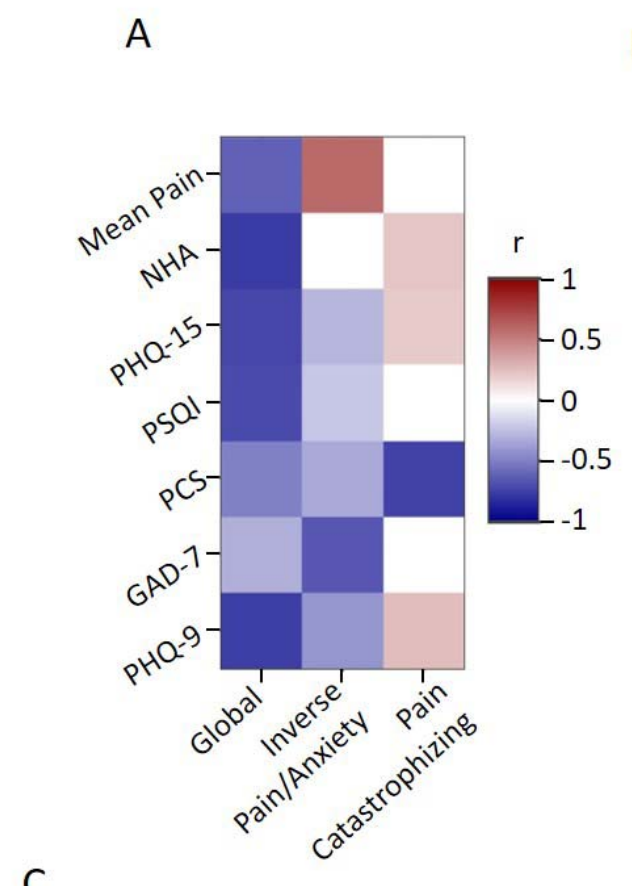

C

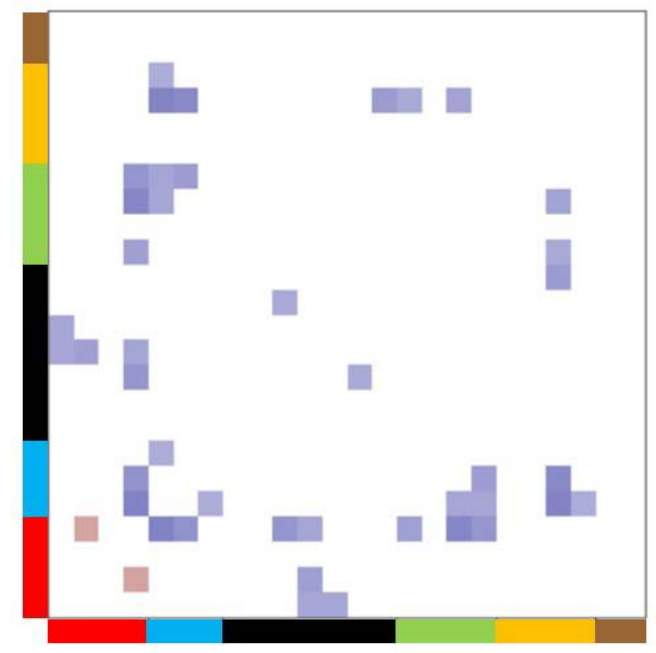

B Default
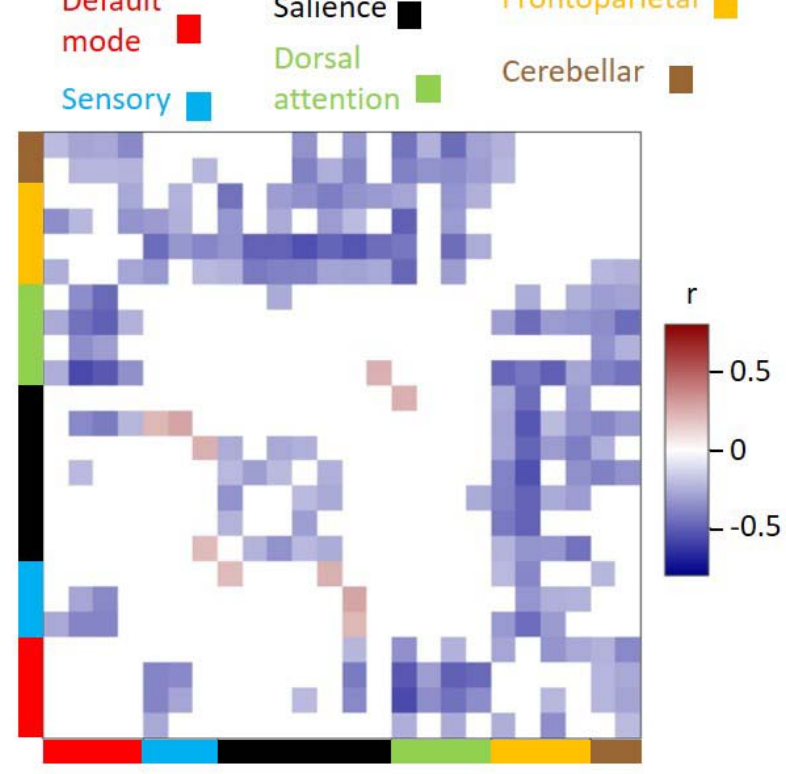

D

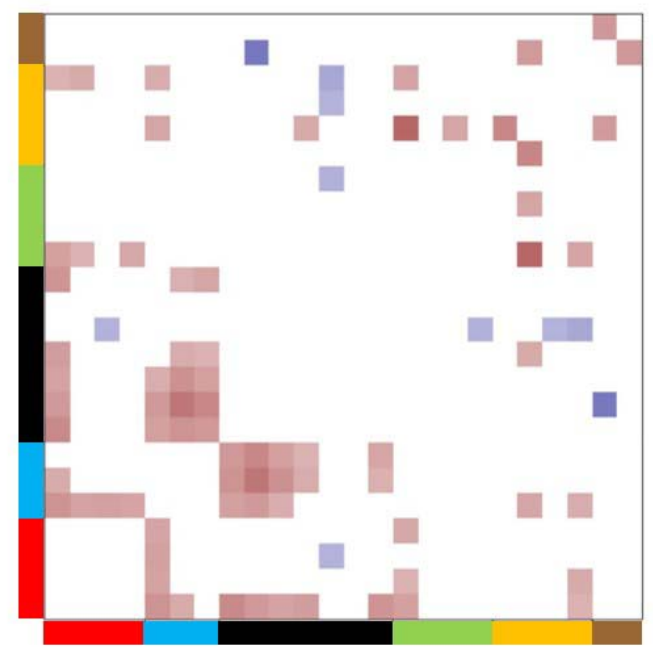

Fig 4. Interpretation of canonical variates. Canonical variates were correlated with input data to facilitate interpretation. A) For clinical data, correlations shown for the three identified dimensions. Correlation between RSFC and global dimension (B), inverse pain/anxiety dimension(C), and pain catastrophizing dimension (D) scores organized over 24 regions of interest in 6 networks. The global dimension was most associated with frontoparietal network and dorsal attention network connectivity with other networks. The inverse pain/anxiety dimension was most related to default mode network and sensorimotor connectivity. The pain catastrophizing dimension was most related to salience, sensorimotor and default mode network connectivity. The same color coding for networks and correlation color range is used in panels B, C, and D. All off-white correlation values are significant for all four panels. Mean Pain=average headache pain; NHA=headache frequency; $\mathrm{PHQ}-15=$ patient healthy questionnaire 15 item; PSQI=Pittsburgh sleep quality index; PCS=pain catastrophizing scale; GAD-7=generalized anxiety disorder; $\mathrm{PHQ}-9=$ patient health questionnaire 9 item; $r=$ Pearson correlation coefficient. 

relationship between average headache pain and anxiety

337 primarily, referred to here as the inverse pain/anxiety

of functional connectivity was quite sparse for this but was

primarily related to anti-correlation between the $\mathrm{DMN}$ and

other networks (Fig 4C). The third symptom canonical variate catastrophizing dimension'.

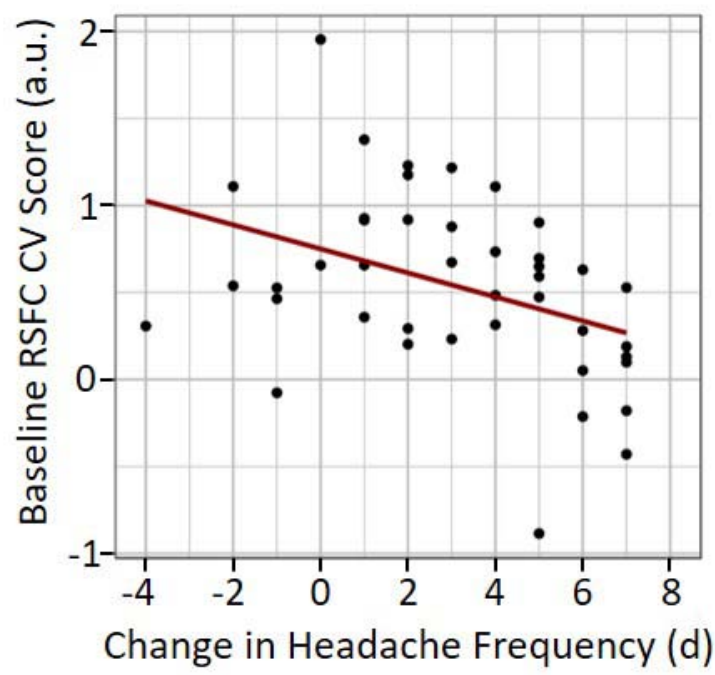

Fig 5. Association between baseline (preintervention) global symptom RSFC canonical variate and change in headache frequency after therapy (positive numbers indicate a reduction in headache days). Subjects with lower scores and more severe symptoms overall had better responses to MBSR+ than subjects were less severe symptoms. RSFC=resting-state functional connectivity; $\mathrm{CV}=$ canonical variate; $\mathrm{d}=$ days.

RSFC Canonical Variates are Associated with Headache Reduction in MBSR+

We sought to determine if RSFC canonical variates were associated with improvements from

global dimension $r_{s}=-0.44, N=42, p=0.0038$ (Fig 5). RSFC canonical variates from neither the inverse 
and chronic ( $\geq 15$ headaches/month) subtypes differs from a multivariate Gaussian distribution. Chronic

and episodic migraine patients were collected at unique sites, creating a potential confound (see

5000 multivariate Gaussian distribution based on the mean and covariance of the 7 clinical measures.

This analysis indicated that the chronic versus episodic clustering was not significantly different from a

374 were other solutions with similar performance ( $k=2$ was best in $23 \%$, no other solutions were

375 consistently as strong; see Fig 6B for silhouette index scores over various values of $\mathrm{k}$ ). To determine

376 significance of a 4 cluster solution, we estimated 5000 multivariate Gaussian distributions based on the

377 mean and covariance of the three RSFC canonical variates. We calculated the silhouette index, a

378 measure of clustering quality, in each of these 5000 distributions and compared the true 4 cluster

379 silhouette index of 0.28 against them (Fig 6C). The true silhouette index of 0.28 was significant relative 

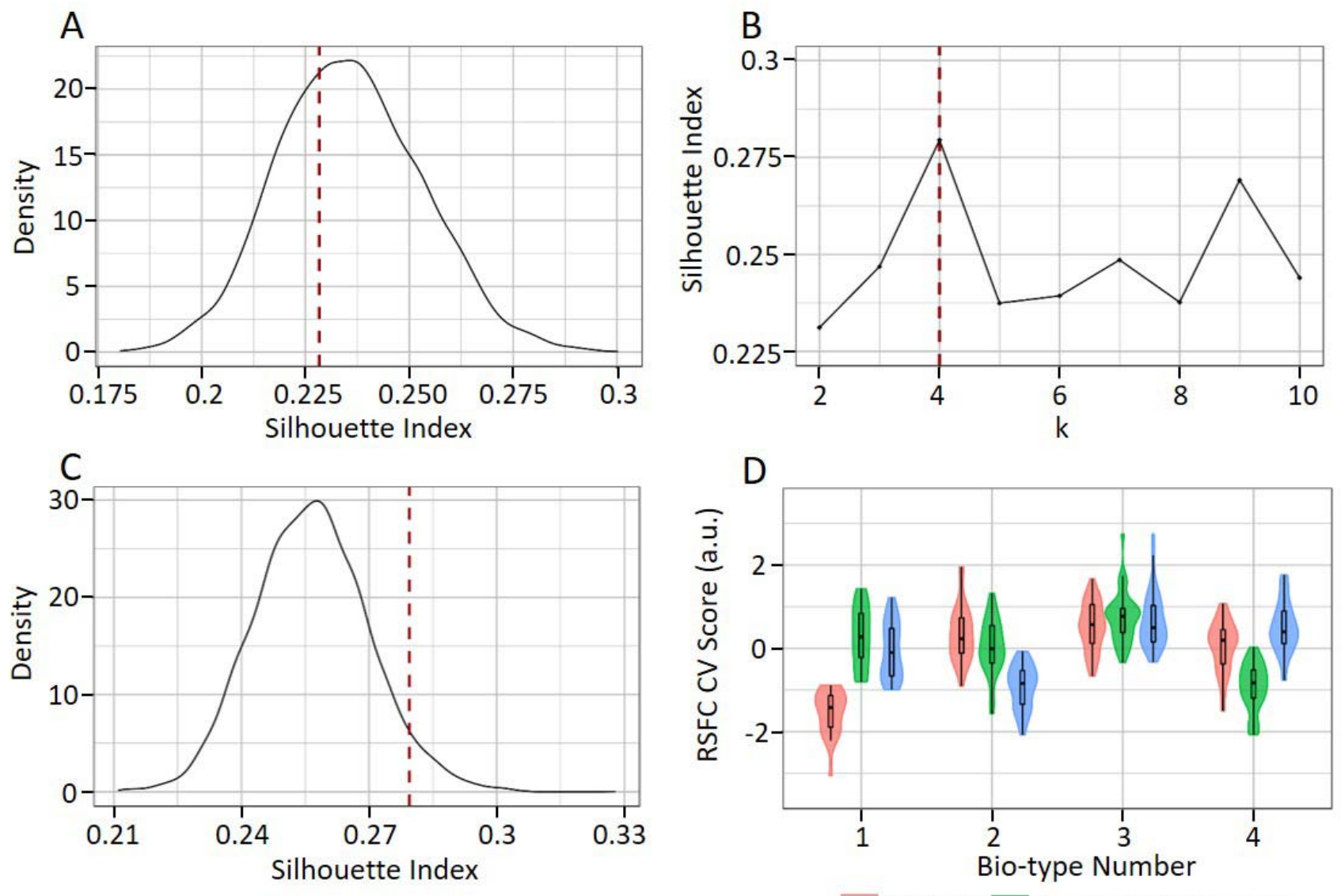

Global Inverse Pain/Anxiety

Pain Catastrophizing

Fig 6. Lack of support for episodic/chronic migraine clustering and identification of a 4 biotype clustering of migraine. A) Lack of significance for clustering migraine into chronic and episodic subtypes based on silhouette index over 5000 multivariate Gaussian distributions of clinical data. B) Silhouette index for various cluster numbers ( $k$ ) from three RSFC canonical variates indicating that $k=4$ had the best performance. C) Significance of clustering into four biotypes based on silhouette index over 5000 multivariate Gaussian distributions of RSFC canonical variate scores. D) Patterns of RSFC canonical variates for three dimensions over the four identified biotypes. Color coding indicates the dimension. Canonical variates are distributed with a mean of zero, therefore negative values indicate below average scores and positive values indicate above average scores. $\mathrm{RSFC}=$ resting-state functional connectivity; $\mathrm{CV}=$ canonical variate; $\mathrm{k}=$ number of clusters.

RSFC canonical variate scores (Fig 6D). Cluster one had negative and therefore below average RSFC

canonical variate values for the global symptom dimension, indicating patients in this group had worse

than average symptoms overall. Cluster two had below average RSFC canonical variate scores for the

pain catastrophizing dimension, indicating patients in this group had above average catastrophizing.

Cluster four had below average pain/anxiety inverse dimension scores, indicating patients in this group 
three dimensions, indicating patients in this group had the opposite pattern of symptoms as all other biotypes.

\section{Discussion}

Migraine is a heterogeneous disorder with variable response to therapy and expression of between-subjects analyses contrasting patients and healthy controls. When variability has been factored in, subjects are often subtyped into categories not derived from biological data that have also not been rigorously tested. When studies do perform within-subjects analyses to associate symptoms with biological data, they often examine only one variable of interest, ignoring the complex relationship

396 between migraine symptoms. In the current study we addressed these existing limitations by using a

397 large sample size, including a wealth of clinical data from well phenotyped subjects, and by relating these clinical profiles to RSFC in a single multivariate statistical step, as opposed to a standard mass univariate analysis. This approach identified three dimensions of covariation between clinical and RSFC data. The first was a global-symptom dimension that was associated with all symptoms and was

401 primarily related to frontoparietal network (FPN) and dorsal attention network (DAN) functional

402 connectivity with other networks. The FPN includes posterior parietal cortex and lateral prefrontal

403 cortex and is consistently associated with cognitive control (Bush \& Shin, 2006; Scolari et al., 2015) as

404 well as acute and chronic pain (Seminowicz et al., 2011; Seminowicz \& Moayedi, 2017; Y. Wang et al., 405 2017). The DAN contains the intraparietal sulcus and frontal eye fields and is consistently involved in 406 attention (Vossel et al., 2014). Our findings suggest that cognitive networks relate to symptoms 407 generally, and that they may function as targets to simultaneously treat all symptoms at once.

408 Consistent with this, the normalization of FPN functional connectivity has been associated with clinical 409 improvement in migraine (Li et al., 2015). The second dimension reflected inverse pain and anxiety 410 scores and was related to default mode (DMN) and sensorimotor network connectivity. The DMN 
411 contains angular gyrus, posterior cingulate cortex, and medial prefrontal cortex and the sensorimotor

412 network contains pre- and postcentral gyri. Finally, the third dimension was a pain catastrophizing

413 dimension and was primarily associated with functional connectivity of salience network (anterior insula

414 and midcingulate cortex) to DMN and sensorimotor networks, and to DMN connectivity with other

415 networks.

We were able to show that the RSFC scores for the global symptom dimension was associated

417 with improvements from the non-pharmacologic MBSR+ treatment of migraine, which has recently been

418 established efficacy for migraine (Seminowicz et al., 2020). Subjects with lower scores and thus more

419 severe symptoms showed a larger reduction in headache frequency following MBSR+ than subjects who

420 had comparatively less severe symptoms. In other words, patients with more severe symptoms overall

421 experienced more pronounced improvements from MBSR+. Additionally, we observed that changes in

422 global dimension RSFC scores towards the positive pole (less symptom severe) were associated with

423 improvements from MBSR+ as well. This might indicate that reducing FPN connectivity to salience,

424 sensorimotor, and dorsal attention networks could enhance the effects of MBSR+, given that the first

425 dimension was negatively associated with this FPN functional connectivity.

The migraine RSFC literature uses small sample sizes and inconsistent analyses, making it

427 difficult to synthesize (Skorobogatykh et al., 2019). However, there do appear to be important points of

428 convergence between our findings and previous work, which further supports the validity of our

429 findings. Abnormal connectivity in migraine is often observed in the FPN, salience, sensorimotor, DMN,

430 and DAN (Hubbard et al., 2014; Jin et al., 2013; Ke et al., 2020; Russo et al., 2012; Xue et al., 2012). We

431 therefore anticipated that these networks would be associated with illness characteristics. Two clinical

432 variables included in our analysis that have been the subject of multiple studies in migraine are

433 headache frequency and pain catastrophizing. Frequency was most related to our first identified global

434 dimension, and associations between headache frequency and functional connectivity of salience and 
FPN nodes (Hubbard et al., 2014; Mainero et al., 2011; Maleki et al., 2012). Pain catastrophizing is often linked to attentional processes (Quartana et al., 2009) and PCS scores are related to anterior insula (key node of the salience network) and sensorimotor connectivity in migraine patients (Hubbard et al., 2014). Our study has major implications to migraine research and clinical trial design. Migraine is currently split into episodic and chronic classifications based on a 15 headache a month cutoff (International Headache Society, 2018).There have been criticisms of the concept of chronic migraine in the literature (Medrea \& Christi, 2018), and in particular over the exact cutoff point between episodic and chronic patients (Torres-Ferrús et al., 2017). We tested the quality of the current two cluster solution for migraine patients in our data based on seven clinical features, finding no evidence against the null hypothesis of a multivariate normal distribution (which does not have clusters). This is especially interesting because the episodic and chronic migraine patient cohorts in our study were collected at two separate sites, which if anything should bias the results toward a chronic versus episodic clustering solution. Additionally, we identified four biotypes of migraine that could be used for predicting therapeutic outcomes in future studies. Patients falling into a given cluster had unique brain-symptom relationships arguing for targeted therapy (Fig 6D). For instance, patients in cluster one had the most extreme global symptom dimension score, which was associated with FPN and DAN connectivity, and perhaps these patients would benefit the most from interventions targeting these networks. On the other hand, cluster two patients who had more extreme pain catastrophizing might benefit the most from interventions that reduce catastrophizing and also target sensorimotor and salience networks. Together, these clustering results argue against the current episodic/chronic clustering that dominates migraine research and clinical trial design, and suggest an alternative based on clinical and biological association.

This study is not without limitations. A major danger when conducting CCA is overfitting, and previously exciting results in depression may have fallen victim to this issue (Dinga et al., 2019). In the 
current study we took several steps to mitigate overfitting. First, we reduced the number of features in the model through parcellations (instead of voxels) for RSFC, feature selection, and PCA. Altogether, this allowed us to greatly increase the ratio of subjects to model features, attenuating overfitting. We took the additional step of performing regularized CCA and tuned lambda parameters based on the held out

463 strength of the first canonical correlation. This resulted in very minimal overfitting for the first canonical correlation. However, overfitting was larger for the second and third canonical correlations, indicating that some caution is warranted when interpreting and using them. Future work could expand on our results by testing this model in new data and benefit from data sharing to create larger sample sizes that 467 are less prone to overfitting. Additionally, statistical inference for clustering is challenging (Dinga et al., 468 2019; Liu et al., 2008). In the current study we tested against a null distribution of a multivariate 469 Gaussian distribution, which when true, would not merit clustering. However, even with rejection of this null hypothesis, it is possible that clustering is still unwarranted because not all multivariate non-

471 Gaussian data are necessarily organized into clusters. Future work could examine the utility of this 472 clustering solution by using the four biotypes to predict therapeutic outcomes from migraine treatment, 473 and by examining if these biotypes exist in additional chronic pain disorders. In conclusion, we identity an association between migraine symptoms - including headache 475 severity, quality of life, affective measures, and coping - and whole-brain resting state functional 476 connectivity, yielding three dimensions of association between these two domains. These results may

477 facilitate the development of personalized medicine, which is limited by treating illnesses as

478 homogeneous groupings. Moreover, the biological association with our clinical data identifies potential

479 targets for therapy and research. Finally, our data argue against the current clustering of migraine 480 patients into chronic and episodic classifications and instead offer an alternative that is grounded in 481 clinical presentation and biology simultaneously. 
Acknowledgements. The authors declare no conflicts of interest. Funding: NCCIH/NIH R01 AT007176 to

DAS; generous gifts from the Wings Foundation, the Wintercreek Foundation, and the Higgins Family

Trust to $\mathrm{BS}$ and $\mathrm{RC}$.

\section{References}

Abraham, A., Pedregosa, F., Eickenberg, M., Gervais, P., Mueller, A., Kossaifi, J., Gramfort, A., Thirion, B., \& Varoquaux, G. (2014). Machine learning for neuroimaging with scikit-learn. Frontiers in Neuroinformatics, 8. https://doi.org/10.3389/fninf.2014.00014

489 Avants, B. B., Epstein, C. L., Grossman, M., \& Gee, J. C. (2008). Symmetric diffeomorphic image 490 registration with cross-correlation: Evaluating automated labeling of elderly and neurodegenerative 491 brain. Medical Image Analysis, 12(1), 26-41. https://doi.org/10.1016/j.media.2007.06.004

492 Behzadi, Y., Restom, K., Liau, J., \& Liu, T. T. (2007). A component based noise correction method 493 (CompCor) for BOLD and perfusion based fMRI. Neuroimage, 37(1), 90-101.

494 Benjamini, Y., Drai, D., Elmer, G., Kafkafi, N., \& Golani, I. (2001). Controlling the false discovery rate in 495 behavior genetics research. Behavioural Brain Research, 125(1-2), 279-284.

496 Breslau, N., Davis, G. C., Schultz, L. R., \& Paterson, E. L. (1994). Migraine and major depression: A longitudinal study. Headache: The Journal of Head and Face Pain, 34(7), 387-393.

498 Breslau, N., Schultz, L. R., Stewart, W. F., Lipton, R. B., Lucia, V. C., \& Welch, K. M. A. (2000). Headache 499 and major depression: Is the association specific to migraine? Neurology, 54(2), 308-308.

500 Bush, G., \& Shin, L. M. (2006). The Multi-Source Interference Task: An fMRI task that reliably activates 501 the cingulo-frontal-parietal cognitive/attention network. Nature Protocols, 1(1), 308-313.

502 Buysse, D. J., Reynolds, C. F., Monk, T. H., Berman, S. R., \& Kupfer, D. J. (1989). The Pittsburgh sleep 503 quality index: A new instrument for psychiatric practice and research. Psychiatry Research, 28(2), 193504 213. https://doi.org/10.1016/0165-1781(89)90047-4

505 Charrad, M., Ghazzali, N., Boiteau, V., Niknafs, A., \& Charrad, M. M. (2014). Package 'nbclust.' Journal of 506 Statistical Software, 61, 1-36.

507 Cox, R. W. (1996). AFNI: Software for Analysis and Visualization of Functional Magnetic Resonance 508 Neuroimages. Computers and Biomedical Research, 29(3), 162-173.

509 https://doi.org/10.1006/cbmr.1996.0014

510 Dinga, R., Schmaal, L., Penninx, B. W. J. H., van Tol, M. J., Veltman, D. J., van Velzen, L., Mennes, M., van 511 der Wee, N. J. A., \& Marquand, A. F. (2019). Evaluating the evidence for biotypes of depression:

512 Methodological replication and extension of Drysdale et al. (2017). Neurolmage: Clinical, 22, 101796.

513 https://doi.org/10.1016/j.nicl.2019.101796 
Drysdale, A. T., Grosenick, L., Downar, J., Dunlop, K., Mansouri, F., Meng, Y., Fetcho, R. N., Zebley, B., Oathes, D. J., \& Etkin, A. (2017). Resting-state connectivity biomarkers define neurophysiological subtypes of depression. Nature Medicine, 23(1), 28-38.

Edvinsson, L., Haanes, K. A., Warfvinge, K., \& Krause, D. N. (2018). CGRP as the target of new migraine therapies-Successful translation from bench to clinic. Nature Reviews Neurology, 14(6), 338-350.

Efron, B. (2000). The bootstrap and modern statistics. Journal of the American Statistical Association, 95(452), 1293-1296.

Esteban, O., Markiewicz, C. J., Goncalves, M., DuPre, E., Kent, J. D., Salo, T., Ciric, R., Pinsard, B., Blair, R. W., Poldrack, R. A., \& Gorgolewski, K. J. (2020). fMRIPrep: A robust preprocessing pipeline for functional MRI. Zenodo. https://doi.org/10.5281/zenodo.4055773

Fonov, V., Evans, A., McKinstry, R., Almli, C., \& Collins, D. (2009). Unbiased nonlinear average ageappropriate brain templates from birth to adulthood. Neurolmage, 47, S102. https://doi.org/10.1016/S1053-8119(09)70884-5

Goadsby, P. J., Reuter, U., Hallström, Y., Broessner, G., Bonner, J. H., Zhang, F., Sapra, S., Picard, H., Mikol, D. D., \& Lenz, R. A. (2017). A controlled trial of erenumab for episodic migraine. New England Journal of Medicine, 377(22), 2123-2132.

Gonzalez, I., Déjean, S., Martin, P., \& Baccini, A. (2008). CCA: An R Package to Extend Canonical Correlation Analysis. Journal of Statistical Software, 23(12). https://doi.org/10.18637/jss.v023.i12

Gorgolewski, K. J., Esteban, O., Ellis, D. G., Notter, M. P., Ziegler, E., Johnson, H., Hamalainen, C., Yvernault, B., Burns, C., Manhães-Savio, A., Jarecka, D., Markiewicz, C. J., Salo, T., Clark, D., Waskom, M., Wong, J., Modat, M., Dewey, B. E., Clark, M. G., ... Ghosh, S. (2017). Nipype: A flexible, lightweight and extensible neuroimaging data processing framework in Python. 0.13.1. Zenodo.

https://doi.org/10.5281/zenodo.581704

Greve, D. N., \& Fischl, B. (2009). Accurate and robust brain image alignment using boundary-based registration. Neurolmage, 48(1), 63-72. https://doi.org/10.1016/j.neuroimage.2009.06.060

Hadjikhani, N., Rio, M. S. del, Wu, O., Schwartz, D., Bakker, D., Fischl, B., Kwong, K. K., Cutrer, F. M., Rosen, B. R., Tootell, R. B. H., Sorensen, A. G., \& Moskowitz, M. A. (2001). Mechanisms of migraine aura revealed by functional MRI in human visual cortex. Proceedings of the National Academy of Sciences, 98(8), 4687-4692. https://doi.org/10.1073/pnas.071582498

Headache Classification Committee of the International Headache Society (IHS) The International Classification of Headache Disorders, 3rd edition. (2018). Cephalalgia, 38(1), 1-211.

https://doi.org/10.1177/0333102417738202

Hotelling, Harold. (1935). Canonical correlation analysis (cca). Journal of Educational Psychology.

Hubbard, C. S., Khan, S. A., Keaser, M. L., Mathur, V. A., Goyal, M., \& Seminowicz, D. A. (2014). Altered brain structure and function correlate with disease severity and pain catastrophizing in migraine patients. Eneuro, 1(1). 
Jenkinson, M., Bannister, P., Brady, M., \& Smith, S. (2002). Improved Optimization for the Robust and Accurate Linear Registration and Motion Correction of Brain Images. Neurolmage, 17(2), 825-841. https://doi.org/10.1006/nimg.2002.1132

Jin, C., Yuan, K., Zhao, L., Zhao, L., Yu, D., von Deneen, K. M., Zhang, M., Qin, W., Sun, W., \& Tian, J. (2013). Structural and functional abnormalities in migraine patients without aura. NMR in Biomedicine, 26(1), 58-64.

Johnson, W. E., Li, C., \& Rabinovic, A. (2007). Adjusting batch effects in microarray expression data using empirical Bayes methods. Biostatistics, 8(1), 118-127. https://doi.org/10.1093/biostatistics/kxj037

Karthik, N., Kulkarni, G. B., Taly, A. B., Rao, S., \& Sinha, S. (2012). Sleep disturbances in 'migraine without aura'-A questionnaire based study. Journal of the Neurological Sciences, 321(1-2), 73-76.

Ke, J., Yu, Y., Zhang, X., Su, Y., Wang, X., Hu, S., Dai, H., Hu, C., Zhao, H., \& Dai, L. (2020). Functional Alterations in the Posterior Insula and Cerebellum in Migraine Without Aura: A Resting-State MRI Study. Frontiers in Behavioral Neuroscience, 14. https://doi.org/10.3389/fnbeh.2020.567588

Kroenke, K., Spitzer, R. L., \& Williams, J. B. (2001). The PHQ-9: Validity of a brief depression severity measure. Journal of General Internal Medicine, 16(9), 606-613.

Kroenke, K., Spitzer, R. L., \& Williams, J. B. W. (2002). The PHQ-15: Validity of a New Measure for Evaluating the Severity of Somatic Symptoms. Psychosomatic Medicine, 64(2), 258-266.

Leonardi, M., \& Raggi, A. (2013). Burden of migraine: International perspectives. Neurological Sciences: Official Journal of the Italian Neurological Society and of the Italian Society of Clinical Neurophysiology, 34 Supp/ 1, S117-118. https://doi.org/10.1007/s10072-013-1387-8

Li, K., Zhang, Y., Ning, Y., Zhang, H., Liu, H., Fu, C., Ren, Y., \& Zou, Y. (2015). The effects of acupuncture treatment on the right frontoparietal network in migraine without aura patients. The Journal of Headache and Pain, 16(1), 33.

Lin, Y.-K., Lin, G.-Y., Lee, J.-T., Lee, M.-S., Tsai, C.-K., Hsu, Y.-W., Lin, Y.-Z., Tsai, Y.-C., \& Yang, F.-C. (2016). Associations between sleep quality and migraine frequency: A cross-sectional case-control study. Medicine, 95(17).

Lipton, R. B., Bigal, M. E., Diamond, M., Freitag, F., Reed, M. L., \& Stewart, W. F. (2007). Migraine prevalence, disease burden, and the need for preventive therapy. Neurology, 68(5), 343-349.

Lipton, R. B., Stewart, W. F., Diamond, S., Diamond, M. L., \& Reed, M. (2001). Prevalence and Burden of Migraine in the United States: Data From the American Migraine Study II. Headache: The Journal of Head and Face Pain, 41(7), 646-657. https://doi.org/10.1046/j.1526-4610.2001.041007646.x

Liu, Y., Hayes, D. N., Nobel, A., \& Marron, J. S. (2008). Statistical significance of clustering for highdimension, low-sample size data. Journal of the American Statistical Association, 103(483), 1281-1293.

Mainero, C., Boshyan, J., \& Hadjikhani, N. (2011). Altered functional magnetic resonance imaging resting-state connectivity in periaqueductal gray networks in migraine. Annals of Neurology, 70(5), 838845 . 
Maleki, N., Becerra, L., Brawn, J., Bigal, M., Burstein, R., \& Borsook, D. (2012). Concurrent functional and structural cortical alterations in migraine. Cephalalgia, 32(8), 607-620.

Mclntosh, A. R. (2000). Towards a network theory of cognition. Neural Networks: The Official Journal of the International Neural Network Society, 13(8-9), 861-870. https://doi.org/10.1016/s08936080(00)00059-9

Medrea, I., \& Christi, S. (2018). Chronic Migraine - Evolution of the Concept and Clinical Implications. Headache: The Journal of Head and Face Pain, 58(9), 1495-1500. https://doi.org/10.1111/head.13380

Mihalik, A., Ferreira, F. S., Rosa, M. J., Moutoussis, M., Ziegler, G., Monteiro, J. M., Portugal, L., Adams, R. A., Romero-Garcia, R., \& Vértes, P. E. (2019). Brain-behaviour modes of covariation in healthy and clinically depressed young people. Scientific Reports, 9(1), 1-11.

Murphy, K., \& Fox, M. D. (2017). Towards a consensus regarding global signal regression for resting state functional connectivity MRI. Neuroimage, 154, 169-173.

Muschelli, J., Nebel, M. B., Caffo, B. S., Barber, A. D., Pekar, J. J., \& Mostofsky, S. H. (2014). Reduction of motion-related artifacts in resting state fMRI using aCompCor. Neuroimage, 96, 22-35.

Noble, S., Scheinost, D., Finn, E. S., Shen, X., Papademetris, X., McEwen, S. C., Bearden, C. E., Addington, J., Goodyear, B., Cadenhead, K. S., Mirzakhanian, H., Cornblatt, B. A., Olvet, D. M., Mathalon, D. H., McGlashan, T. H., Perkins, D. O., Belger, A., Seidman, L. J., Thermenos, H., ... Constable, R. T. (2017). Multisite reliability of MR-based functional connectivity. Neurolmage, 146, 959-970. https://doi.org/10.1016/j.neuroimage.2016.10.020

Petrovic, S. (2006). A comparison between the silhouette index and the davies-bouldin index in labelling ids clusters. Proceedings of the 11th Nordic Workshop of Secure IT Systems, 2006, 53-64.

Pituch, K. A., \& Stevens, J. P. (2015). Applied multivariate statistics for the social sciences: Analyses with SAS and IBM'S SPSS. Routledge.

Power, J. D., Barnes, K. A., Snyder, A. Z., Schlaggar, B. L., \& Petersen, S. E. (2012). Spurious but systematic correlations in functional connectivity MRI networks arise from subject motion. Neuroimage, 59(3), 2142-2154. https://doi.org/10.1016/j.neuroimage.2011.10.018

Power, J. D., Mitra, A., Laumann, T. O., Snyder, A. Z., Schlaggar, B. L., \& Petersen, S. E. (2014). Methods to detect, characterize, and remove motion artifact in resting state fMRI. Neurolmage, 84, 320-341. https://doi.org/10.1016/j.neuroimage.2013.08.048

Power, J. D., Plitt, M., Laumann, T. O., \& Martin, A. (2017). Sources and implications of whole-brain fMRI signals in humans. Neuroimage, 146, 609-625.

Pruim, R. H. R., Mennes, M., van Rooij, D., Llera, A., Buitelaar, J. K., \& Beckmann, C. F. (2015). ICAAROMA: A robust ICA-based strategy for removing motion artifacts from fMRI data. Neurolmage, 112, 267-277. https://doi.org/10.1016/j.neuroimage.2015.02.064

Quartana, P. J., Campbell, C. M., \& Edwards, R. R. (2009). Pain catastrophizing: A critical review. Expert Review of Neurotherapeutics, 9(5), 745-758. 
622

623

624

625

626

627

628

629

630

631

632

633

634

635

636

637

638

639

640

641

642

643

644

645

646

647

648

649

650

651

652

653

654

655

656

657

658

659

Rasmussen, B. K. (1995). Epidemiology of migraine. Biomedicine \& Pharmacotherapy = Biomedecine \& Pharmacotherapie, 49(10), 452-455. https://doi.org/10.1016/0753-3322(96)82689-8

Russo, A., Tessitore, A., Giordano, A., Corbo, D., Marcuccio, L., De Stefano, M., Salemi, F., Conforti, R., Esposito, F., \& Tedeschi, G. (2012). Executive resting-state network connectivity in migraine without aura. Cephalalgia, 32(14), 1041-1048.

Schwedt, T. J., Chiang, C.-C., Chong, C. D., \& Dodick, D. W. (2015). Functional MRI of migraine. The Lancet Neurology, 14(1), 81-91.

Scolari, M., Seidl-Rathkopf, K. N., \& Kastner, S. (2015). Functions of the human frontoparietal attention network: Evidence from neuroimaging. Current Opinion in Behavioral Sciences, 1, 32-39.

Seminowicz, D. A., Burrowes, S. A. B., Kearson, A., Zhang, J., Krimmel, S. R., Samawi, L., Furman, A. J., Keaser, M. L., Gould, N. F., Magyari, T., White, L., Goloubeva, O., Goyal, M., Peterlin, B. L., \& Haythornthwaite, J. A. (2020a). Enhanced mindfulness-based stress reduction in episodic migraine: A randomized clinical trial with magnetic resonance imaging outcomes. PAIN, 161(8), 1837-1846. https://doi.org/10.1097/j.pain.0000000000001860

Seminowicz, D. A., Burrowes, S. A. B., Kearson, A., Zhang, J., Krimmel, S. R., Samawi, L., Furman, A. J., Keaser, M. L., Gould, N. F., Magyari, T., White, L., Goloubeva, O., Goyal, M., Peterlin, B. L., \& Haythornthwaite, J. A. (2020b). Enhanced mindfulness-based stress reduction in episodic migraine: A randomized clinical trial with magnetic resonance imaging outcomes. PAIN, 161(8), 1837-1846. https://doi.org/10.1097/j.pain.0000000000001860

Seminowicz, D. A., \& Moayedi, M. (2017). The dorsolateral prefrontal cortex in acute and chronic pain. The Journal of Pain, 18(9), 1027-1035.

Seminowicz, D. A., Wideman, T. H., Naso, L., Hatami-Khoroushahi, Z., Fallatah, S., Ware, M. A., Jarzem, P., Bushnell, M. C., Shir, Y., \& Ouellet, J. A. (2011). Effective treatment of chronic low back pain in humans reverses abnormal brain anatomy and function. Journal of Neuroscience, 31(20), 7540-7550.

Skorobogatykh, K., van Hoogstraten, W. S., Degan, D., Prischepa, A., Savitskaya, A., Ileen, B. M., Bentivegna, E., Skiba, I., D’Acunto, L., Ferri, L., Sacco, S., Hansen, J. M., Amin, F. M., \& European Headache Federation School of Advanced Studies (EHF-SAS). (2019). Functional connectivity studies in migraine: What have we learned? The Journal of Headache and Pain, 20(1), 108.

https://doi.org/10.1186/s10194-019-1047-3

Smith, S. M., Nichols, T. E., Vidaurre, D., Winkler, A. M., Behrens, T. E., Glasser, M. F., Ugurbil, K., Barch, D. M., Van Essen, D. C., \& Miller, K. L. (2015). A positive-negative mode of population covariation links brain connectivity, demographics and behavior. Nature Neuroscience, 18(11), 1565-1567.

Spitzer, R. L., Kroenke, K., Williams, J. B., \& Löwe, B. (2006). A brief measure for assessing generalized anxiety disorder: The GAD-7. Archives of Internal Medicine, 166(10), 1092-1097.

Sullivan, M. J., Bishop, S. R., \& Pivik, J. (1995). The pain catastrophizing scale: Development and validation. Psychological Assessment, 7(4), 524.

Tabachnick, B. G., Fidell, L. S., \& Ullman, J. B. (2007). Using multivariate statistics (Vol. 5). Pearson Boston, MA. 
660

661

662

663

664

665

666

667

668

669

670

671

672

673

674

675

676

677

678

679

680

681

682

683

684

685

686

687

688

689

690

691

692

693

694

Torres-Ferrús, M., Quintana, M., Fernandez-Morales, J., Alvarez-Sabin, J., \& Pozo-Rosich, P. (2017). When does chronic migraine strike? A clinical comparison of migraine according to the headache days suffered per month. Cephalalgia, 37(2), 104-113. https://doi.org/10.1177/0333102416636055

Tustison, N. J., Avants, B. B., Cook, P. A., Zheng, Y., Egan, A., Yushkevich, P. A., \& Gee, J. C. (2010). N4ITK: Improved N3 Bias Correction. IEEE Transactions on Medical Imaging, 29(6), 1310-1320.

https://doi.org/10.1109/TMI.2010.2046908

Van Horn, J. D., \& Toga, A. W. (2009). Multi-Site Neuroimaging Trials. Current Opinion in Neurology, 22(4), 370-378. https://doi.org/10.1097/WCO.0b013e32832d92de

Vossel, S., Geng, J. J., \& Fink, G. R. (2014). Dorsal and ventral attention systems: Distinct neural circuits but collaborative roles. The Neuroscientist, 20(2), 150-159.

Wang, H.-T., Poerio, G., Murphy, C., Bzdok, D., Jefferies, E., \& Smallwood, J. (2018). Dimensions of experience: Exploring the heterogeneity of the wandering mind. Psychological Science, 29(1), 56-71.

Wang, H.-T., Smallwood, J., Mourao-Miranda, J., Xia, C. H., Satterthwaite, T. D., Bassett, D. S., \& Bzdok, D. (2020). Finding the needle in a high-dimensional haystack: Canonical correlation analysis for neuroscientists. Neurolmage, 216, 116745.

Wang, Y., Cao, D., Remeniuk, B., Krimmel, S., Seminowicz, D. A., \& Zhang, M. (2017). Altered brain structure and function associated with sensory and affective components of classic trigeminal neuralgia. Pain, 158(8), 1561-1570.

Whitfield-Gabrieli, S., \& Nieto-Castanon, A. (2012). Conn: A functional connectivity toolbox for correlated and anticorrelated brain networks. Brain Connectivity, 2(3), 125-141.

Xia, C. H., Ma, Z., Ciric, R., Gu, S., Betzel, R. F., Kaczkurkin, A. N., Calkins, M. E., Cook, P. A., de la Garza, A. G., \& Vandekar, S. N. (2018). Linked dimensions of psychopathology and connectivity in functional brain networks. Nature Communications, 9(1), 1-14.

Xue, T., Yuan, K., Zhao, L., Yu, D., Zhao, L., Dong, T., Cheng, P., von Deneen, K. M., Qin, W., \& Tian, J. (2012). Intrinsic brain network abnormalities in migraines without aura revealed in resting-state fMRI. Plos One, 7(12), e52927.

Yeo, B. T., Krienen, F. M., Sepulcre, J., Sabuncu, M. R., Lashkari, D., Hollinshead, M., Roffman, J. L., Smoller, J. W., Zöllei, L., \& Polimeni, J. R. (2011). The organization of the human cerebral cortex estimated by intrinsic functional connectivity. Journal of Neurophysiology.

Yu, M., Linn, K. A., Cook, P. A., Phillips, M. L., Mclnnis, M., Fava, M., Trivedi, M. H., Weissman, M. M., Shinohara, R. T., \& Sheline, Y. I. (2018). Statistical harmonization corrects site effects in functional connectivity measurements from multi-site fMRI data. Human Brain Mapping, 39(11), 4213-4227. https://doi.org/10.1002/hbm.24241

Yuan, H., Spare, N. M., \& Silberstein, S. D. (2019). Targeting cgrp for the prevention of migraine and cluster headache: A narrative review. Headache: The Journal of Head and Face Pain, 59, 20-32. 
bioRxiv preprint doi: https://doi.org/10.1101/2021.03.31.437905; this version posted April 1, 2021. The copyright holder for this preprint (which was not certified by peer review) is the author/funder, who has granted bioRxiv a license to display the preprint in perpetuity. It is made available under aCC-BY-NC-ND 4.0 International license.

695 Zhang, Y., Brady, M., \& Smith, S. (2001). Segmentation of brain MR images through a hidden Markov 696 random field model and the expectation-maximization algorithm. IEEE Transactions on Medical Imaging, 697 20(1), 45-57. https://doi.org/10.1109/42.906424 